\title{
SATELLITE OBSERVATIONS OF THE NITRIC OXIDE NIGHTGLOW
}

\author{
Jean-Claude Gerard* \\ Institut d'Astrophysique \\ Universite de Liege, B4200-Ougree, Belgium
}

\begin{abstract}
The No $\delta$-bands have been observed photometrically by the ultraviolet telescope onboard the TD-1 ESRO satellite. The maximum intensity for the system is about $220 \mathrm{R}$, in good agreement with a previous rocket measurement.

Assuming that the excitation is due to the $\mathrm{N}+\mathrm{O} \rightarrow \mathrm{NO}\left(\mathrm{C}^{2} \Pi, \mathrm{V}=0\right)$ preas sociation, we used the observed profile to derive the atomic nitrogen density distribution. We found a peak of about $9 \times 107 \mathrm{~cm}-3$ at about $180 \mathrm{~km}$. A comparison is made with theoretical predictions, and some implications of such a high $N\left({ }^{4} S\right)$ density are discussed.
\end{abstract}

The No $\delta$-bands have been observed in the nightglow spectrum by balloon-borne (Cohen-Sabbin and Vuillemin, 1973) and rocket-borne ultraviolet spectrometers (Feldman and Takacs, 1974). The first set of observations clearly revealed the presence of the $(0,1),(0,2)$, and $(0,3)$ No $\delta$-bands, but no absolute intensity or vertical distribution has been deduced. The rocket spectra showed features from the No $\delta$ - and $\gamma$-systems, but the geometry of the observations was complicated by resonance scattering of the $\gamma$-bands above a shadow altitude of $215 \mathrm{~km}$. The excitation of the NO(C2 II) state was attributed to the chemiluminescent preassociation of $\mathrm{N}$ and $\mathrm{O}$ in the reaction:

$$
\mathrm{N}+\mathrm{O} \rightarrow \mathrm{NO}\left(\mathrm{c}^{2} \Pi, \mathrm{v}=0\right)
$$

Photometric measurements of the altitude distribution of the ultraviolet nightglow emission were obtained by the UV telescope onboard the ESRO TD-1A satellite in December 1973 and January 1974. During this period in a hibernation mode, the satellite spun about its sun-stabilized axis at a rate of 1.89 degrees/sec. Consequently, the optical axis of the telescope scanned a plane parallel to the terminator. The experiment was turned on at high latitude in the dark sector and turned off when the plane of observation approached the terminator. One of the

*"Aspirant" of the Belgian National Foundation for Scientific Research (NRS). Now at the Laboratory for Atmospheric and Space Physics, University of Colorado, Boulder, 'Colorado 80302 .

Copyright 1975 by the American Geophysical Union. channels, labelled $A_{3}$, recorded a weak signal when the line of sight scanned close to the horizon. This emission is attributed to the No $\delta$-bands. Fig. 1 displays three such scans recorded on 16 and 17 December 1973 at a spacecraft altitude of $540 \mathrm{~km}$ and at various latitudes. The altitude of the line of sight is indicated at the top of the figure. The solar zenith angle varies from $110: 6$ to $97: 7$. The dotted lines show the level of the background noise. In the

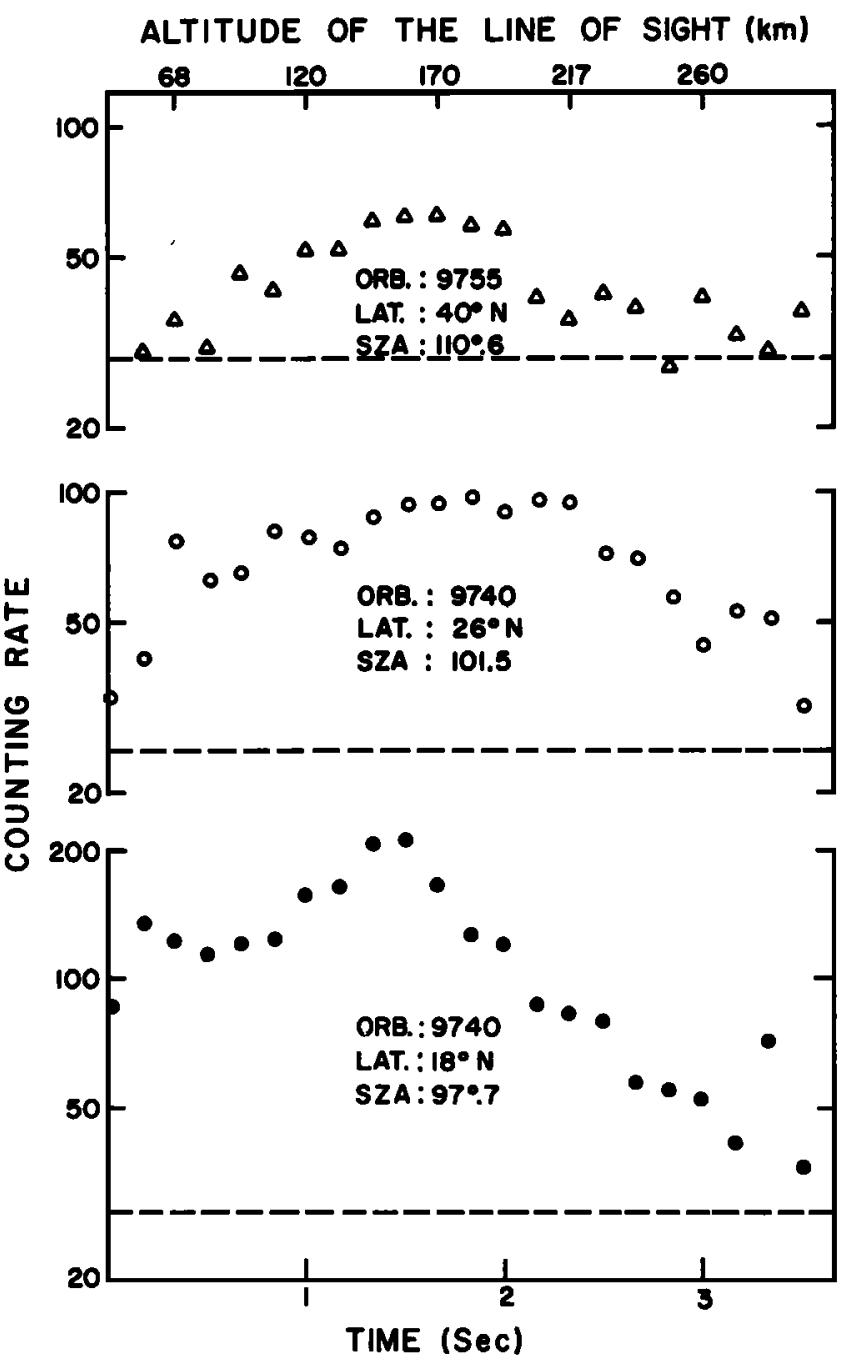

Fig. 1. Example of three horizon scans by the TD-1 UV telescope at various solar zenith angles (SZA). The dots indicate the number of counts/0.16 sec measured as a function of time. 
following, we shail concentrate on the first example for which the solar depression is large enough to prevent any contribution from the No $\gamma$-resonance scattering in the passband (Fig. 2).

The spectral response of the $\mathrm{A}_{3}$ channel on a diffuse source (Boksenberg and Gerard, 1973) is a rough1y rectangular function between 1750 and $2150 \mathrm{~A}$. The sampling rate of the photon counting is $6 \mathrm{sec}^{-1}$. The counting rate can be converted into absolute emission rate of the $\delta$-system provided the spectral distribution of the intensity is known. Table 1 gives the branching ratios as calculated using a $q^{\prime}{ }^{\prime \prime},{ }^{3}$ dependence with Franck-Condon factors from Ory (1964). They are compared with those deduced from high resolution recombination spectra obtained in the laboratory by Kley (1973) and with the airglow spectrum of Feldman and Takacs. The agreement among the three sets of intensities is satisfactory, and an instrument sensitivity of $6.1 \mathrm{R} /$ count in the channel is deduced for the whole $\delta$-system. A maximum intensity of about $220 \mathrm{R}$ is thus observed near $165 \mathrm{~km}$ and the corresponding emission rate of the $(0,1)$ band is on the order of $50 \mathrm{R}$. This vaiue compares favorably with the $83 \mathrm{R}$ measured by Feldman and Takacs for this band. The $v^{T}=0$ progression of the $\gamma$-bands is excited in the laboratory recombination spectrum. The $\mathrm{v}^{\prime}=3$ progression is very weak (Kley, 1973), and, consequently there is negligible contribution from this system in channe1 $\mathrm{A}_{3}$. The weak emission rate factor of the $\delta$-bands (Barth, 1966), combined with the low abundance of NO above the shadow height, gives a negligible contribution of resonance scattering to the observed signal.

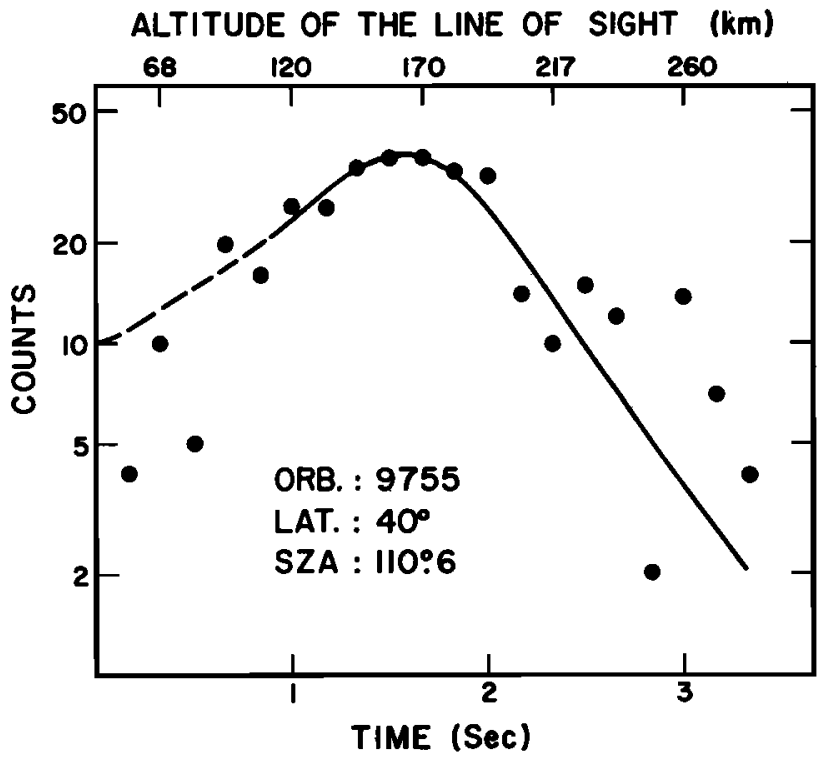

Fig. 2. Example of a horizon scan with background subtracted. The solid line is a fit to the data.
The apparent emission rate $4 \pi \phi$ observed for an altitude $z_{0}$ of the line of sight is related to the volume emission rate, $n$, by the relationship:

$$
4 \pi \phi_{z_{0}}=2 \int_{z_{0}}^{\infty} n(s) d s
$$

where $s$ is the variable along the line of sight. Integral (2) must be inverted to determine the altitude distribution of $n$. For this purpose, a four-parameter analytical function of the form

$$
\begin{aligned}
\mathrm{F}_{z}\left(\mathrm{H}_{1}, \mathrm{H}_{2}, \mathrm{z}_{\mathrm{M}}, \mathrm{A}\right)= & \frac{\mathrm{A}}{\exp \left[\left(\mathrm{z}-\mathrm{z}_{\mathrm{M}}\right) / \mathrm{H}_{1}\right]+\exp \left[-\left(z-z_{M}\right) / H_{2}\right]}
\end{aligned}
$$

was assumed to represent adequately $n(z)$. The parameters were determined so that integral (2), with $\mathrm{F}_{\mathrm{z}}$ as the integrand, fit the observed profile. This fit is illustrated in Fig. 2; a good agreement is not expected at low altitudes where the atmosphere is optically thick in that spectral range.

If we assume that reaction (1) is responsible for the production of the NO $\left(C^{2} I\right)$ state, the atomic nitrogen density profile is given by:

$$
[\mathrm{N}]=\frac{\mathrm{F}_{z}}{\mathrm{k}_{\delta}[\mathrm{O}]},
$$

where $k$ is the total rate coefficient of reaction (1), for which recent laboratory measurements (Mandelman et al., 1973) give

\begin{tabular}{|c|c|c|c|c|}
\hline \multirow{2}{*}{$\begin{array}{l}\text { Trans- } \\
\text { ition }\end{array}$} & \multirow[b]{2}{*}{$\lambda(\mathrm{A})$} & \multicolumn{3}{|c|}{ Branching Ratios } \\
\hline & & $q_{v^{\prime} v^{\prime \prime} v^{3}}$ & Lab & Airglow \\
\hline $0-0$ & 1912 & 0.19 & 0.16 & 0.17 \\
\hline $0-1$ & 1983 & 0.29 & 0.22 & 0.25 \\
\hline $0-2$ & 2058 & 0.24 & 0.22 & 0.18 \\
\hline $0-3$ & 2138 & 0.15 & 0.17 & \\
\hline $0-4^{a}$ & 2223 & 0.08 & 0.12 & \\
\hline $0-5^{a}$ & 2314 & 0.035 & 0.06 & \\
\hline $0-6^{a}$ & 2407 & 0.015 & 0.03 & \\
\hline $0-7^{a}$ & & & 0.015 & \\
\hline $0-8^{a}$ & & & 0.005 & \\
\hline
\end{tabular}
a value of $1.5 \times 10^{-17} \mathrm{~cm}^{3} \mathrm{sec}^{-1}$. A weak

TABLE 1

Intensity distribution of the NO $\delta$-bands

autside the channel passband. 
temperature dependence of $k$ (25\% from $300^{\circ} \mathrm{K}$ to $600^{\circ} \mathrm{K}$ ) has been heglected here. The result of this calculation is illustrated in Fig. 3 where the atomic oxygen density is taken from CIRA (1972) for an exospheric temperature of $800^{\circ} \mathrm{K}$, calculat for the day of observation. The curve
gives a maximum density of about $9 \times 10^{7}$ $\mathrm{cm}^{-3}$ near $180 \mathrm{~km}$. The gradient below $150 \mathrm{~km}$ is very steep and is determined by chemical reactions.

The intensity of the signal observed is in agreement with previous spectral observations. Its altitude distribution provides a method to determine the atomic nitrogen profile in the nighttime atmosphere provided reaction (1) only contributes to the excitation of the No $\delta$-bands. Other similar data to be analyzed will provide information on the latitude dependence of the $\mathrm{N}$ density. The case illustrated in Fig. 3 corresponds to one of the brightest scans and the deduced $N$ density must probably be considered as an upper limit.

In the range of altitude considered here, $N(4 S)$ atoms are produced by various reactions and mainly destroyed by the process :

$$
\mathrm{N}\left({ }^{4} \mathrm{~S}\right)+\mathrm{NO} \rightarrow \mathrm{N}_{2}+\mathrm{O},
$$

whose rate coefficient is $1.5 \times 10^{-12} \sqrt{\mathrm{T}}$ (Phillips and Schiff, 1962; Nicolet, 1965). Reaction (4) is also the dominant destruction mechanism for nitric oxide below $250 \mathrm{~km}$. Consequently, the abundance of $N(4 S)$ depends on the NO density and vice-versa.

The experimental profile of Fig. 3 can be compared with recent models for the $\mathrm{N}(4 \mathrm{~S})$ and NO distributions. Strobel's (1971) model at $18.30 \mathrm{~L} . \mathrm{T}$. predicts a maximum density [N] $\sim 5 \times 106 \mathrm{~cm}^{-3}$ at an altitude of $240 \mathrm{~km}$ with an NO density of $4 \times 10^{6} \mathrm{~cm}-3$. A comparison of the NO, $\mathrm{N}(2 \mathrm{D})$ and the densities of major neutrals

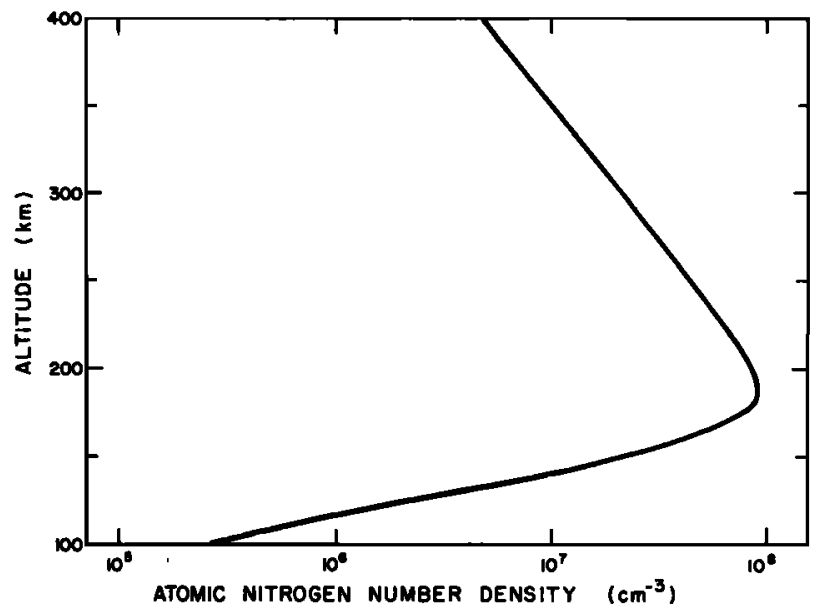

Fig. 3. Atomic nitrogen density profile deduced from the NO $\delta$-bands intensity by inverting the solid curve of Fig. 2 . and ions locally measured by the Atmosphere Explorer C satellite was recently made by Rusch et al. (1975). They predict high quantities of $\mathrm{N}(4 \mathrm{~S})\left(\simeq 1 \times 10^{8}\right.$ $\mathrm{cm}^{-3}$ at $180 \mathrm{~km}$ ), coupled with low No dabundance $\left(\simeq 106^{\prime} \mathrm{cm}^{-3}\right)$, in very good agreement with these measurements.

High atomic nitrogen densities are expected to have the following conse-

quences: $\mathrm{A}$ large density of $\mathrm{N}\left({ }^{4} \mathrm{~S}\right)$ at early night gives very short No lifetimes against reaction (4). Consequently, below $280 \mathrm{~km}$ the nitric oxide densitites can be expected to drop drastically very soon after sunset.

2. Coupling our calculation for $[\mathrm{N}]$ at $160 \mathrm{~km}$ with NO measurements by Rusch et a1., [NO] $\simeq 5 \times 10^{6} \mathrm{~cm}^{-3}$, we found a production rate $\simeq 1 \times 10^{4}$ $\mathrm{sec}^{-1}$ for NO, which is somewhat more than the upper limit predicted by models. However, the uncertainties of $\mathrm{N}$ and No densities rule out an accurate comparison until simultaneous measurements of NO, $N\left({ }^{4} \mathrm{~S}\right)$ and $\mathrm{N}(2 \mathrm{D})$ can be made.

3. Any analysis of the $\lambda 1200 \mathrm{~A}$ dayglow profile must take radiation trapping into account for such high $\mathrm{N}\left({ }^{4} \mathrm{~S}\right)$ densities.

4. The density of $\mathrm{N}^{+}$ions has been extensively measured and can be used to determine whether the amount of atomic nitrogen deduced here is consistent with the $\mathrm{N}^{+}$density. The analysis of the $\mathrm{N}^{+}$distribution may also provide information on the $\mathrm{N}$ density.

More detailed discussion of the implication of high [N] values can be done when the variability of the amount and vertical distribution of atomic nitrogen is known. A direct simultaneous measurement of $[\mathrm{N}]$ and other constituents would also be most useful to clarify the $\mathrm{N}$-NO reaction scheme. Work along these lines is in progress.

Acknowledgments. We acknowledge with thanks C. Barth, I. Stewart, and P. Feldman for their critical reading of the manuscript. C. Chisholm and A. Tilkin were most helpful with the data processing. We thank the TD-1 team and the European Space Operation Centre (ESOC) which coordinated the telemetry operations. A NATO post-doctoral fellowship has partially sponsored the author's stay at LASP.

\section{References}

Barth, C. A., "The ultraviolet spectroscopy of planets," in The middle ultraviolet: Its science and technology, pp. 186-189, John Wiley and Sons, 1966 .

Boksenberg, A., and J. C. Gerard, U1traviolet observations of equatorial 
dayglow above the $F_{2}$ peak, J. Geophys. Res., 78, 4641, 1973.

CIRA, COSPAR Internationa1 Reference Atmosphere, pp. 302-303, Akademie Verlag, Berlin, 1972.

Cohen-Sabbin, J., and A. Vuillemin, Ultraviolet nightglow spectrum from $1900 \mathrm{~A}$ to $3400 \mathrm{~A}$, Astrophys. Space Sci., 24, $127,1973$.

Feldman, P. D., and P. Z. Takacs, Nitric oxide gamma and delta band emission at twilight, Geophys. Res. Let., 1, $169,1974$.

Kley, D., Mechanism der $\mathrm{N}+0$ rekombination bei kleinen drucken, pp. 37-46, Habilitationsschrift, Bonn, 1973.

Mandelman, M., T. Carrington, and R. A. Young, Predissociation and its inverse, using resonance absorption NO $\left(\mathrm{C}^{2} \Pi\right) \neq \mathrm{N}+0$, J. Chem. Phys. , $\underline{58}$, 84,1973 .

Nicolet, M., Nitrogen oxides in the chem- osphere, J. Geophys. Res., 70, 679, 1965.

Ory, H. A., Franck-Condon factors and electronic oscillator strengths for nitric oxide ultraviolet band systems, J. Chem. Phys., 40, 562, 1964.

Phillips, L. F., and H. I'. Schiff, Mass spectrometer studies of atom reactions, 1 , Reactions in the atomic nitrogen-ozone system, J. Chem. Phys., 36, 1509, 1962 .

Rusch, D. W. A. I. Stewart, P. B. Hays, and J. H. Hoffman, The NI (5200 A) dayglow, J. Geophys. Res., $\underline{80}$, in press, 1975 .

Strobel, D. F., Diurnal variation of nitric oxide in the upper atmosphere, J. Geophys Res. , 76, 2441, 1971 .

(Received March 14, 1975; accepted March 14, 1975.) 